\title{
High-affinity free ubiquitin sensors as quantitative probes of ubiquitin homeostasis and deubiquitination
}

Yun-Seok Choi ${ }^{1}$, Sarah A. Bollinger ${ }^{1}$, Luisa F. Prada ${ }^{1}$, Francesco Scavone ${ }^{1}$, Tingting $\mathrm{Yao}^{1}$, and Robert E. Cohen ${ }^{1 *}$

${ }^{1}$ Department of Biochemistry \& Molecular Biology, Colorado State University, Fort Collins, Colorado, U.S.A. 80523

* Corresponding author

Address correspondence to:

Robert E. Cohen

Department of Biochemistry \& Molecular Biology

Colorado State University

Fort Collins, CO 80523-1870

Email: bob.cohen@colostate.edu Tel: 970-492-4117 


\begin{abstract}
Ubiquitin (Ub) conjugation is an essential post-translational modification that affects nearly all proteins in eukaryotes. The functions and mechanisms of ubiquitination are areas of extensive and ongoing study, and yet the dynamics and regulation of even free (i.e., unconjugated) Ub are poorly understood. A major impediment has been the lack of simple and robust techniques to quantify Ub levels in cells and to monitor Ub release from conjugates. Here we describe the development of avidity-based fluorescent sensors that address this need. The sensors bind specifically to free $\mathrm{Ub}$, have $K_{d}$ values down to $60 \mathrm{pM}$, and, in concert with a newly developed workflow, allow us to distinguish and quantify the pools of free, protein-conjugated, and thioesterified forms of Ub from cell lysates. Alternatively, free Ub in fixed cells can be visualized microscopically by staining with a sensor. Real-time assays using the sensors afford unprecedented flexibility and precision to measure deubiquitination of virtually any (poly) Ub conjugate.
\end{abstract}




\section{Introduction}

In ubiquitination, free $\mathrm{Ub}$ is activated by formation of a C-terminal thioester first with an E1 Ub-activating enzyme and then an E2 Ub-conjugating enzyme before it is transferred to substrates, usually to form an isopeptide bond with a protein lysine $\varepsilon$-amine ${ }^{1}$. Deubiquitinating enzymes (DUBs) can disassemble Ub-protein conjugates and recycle $\mathrm{Ub}$; thus, cells contain three classes of $\mathrm{Ub}$ : free, thioester-activated, and (iso)peptide conjugated. Because ubiquitination contributes to the regulation of nearly every cellular process, the availability of free Ub needs to be tightly controlled to maintain cell fitness. Mechanisms contributing to $\mathrm{Ub}$ homeostasis include its expression as Ub-protein fusions with ribosome subunits and as polyubiquitin, processing of these precursors to generate free monoUb, and finally recycling from Ub-protein conjugates by DUBs ${ }^{2}$. Perturbations of these processes can deplete cellular free $\mathrm{Ub}$ and cause defects in cell development or neuronal functions ${ }^{2-9}$ and inhibit proliferation in several cancer cell lines ${ }^{10,11}$. Conversely, transgenic mice that overexpress Ub by just 2 or 3 -fold exhibit neurological abnormalities ${ }^{12}$.

Although the need to maintain and regulate intracellular free $\mathrm{Ub}$ is now well established, studies of Ub levels have been hampered by the lack of reagents for specific and quantitative measurements of distinct $U b$ pools. One approach to monitor intracellular $\mathrm{Ub}$ has been ectopic expression of GFP-tagged versions of $\mathrm{Ub}^{13}$, but interpretations of results from such experiments can be compromised by perturbations to the regulation of endogenous $\mathrm{Ub}$ and non-physiological behavior of the tagged $\mathrm{Ub}$. Typically, to quantify endogenous free, conjugated, or total $\mathrm{Ub}$, anti-Ub antibodies in conjunction with ELISA or SDS-PAGE and immunoblotting are used. However, with respect to sensitivity and reliability of quantitation, those approaches have major drawbacks. For example, due to the extremely high structural diversity of polyUb and $\mathrm{Ub}$-protein conjugates ${ }^{14}$, even with monoclonal anti-Ub antibodies, binding efficiencies will vary among the many different forms of $\mathrm{Ub}$ in cell lysates. Moreover, particularly with quantitation by western blots, dynamic range is inherently very limited and reproducibility can be difficult to achieve. More recently, Ub Protein Standard Absolute Quantification (Ub-PSAQ) mass spectrometry (MS) has been described to quantify free and conjugated Ub from cell 
lysates ${ }^{15}$. However, this method does not resolve the pool of thioester-activated Ub, and its dependence on sophisticated instrumentation and sequential affinity-based isolation steps makes it challenging to implement for most laboratories. Finally, none of the aforementioned approaches to $\mathrm{Ub}$ quantitation are amenable to real-time measurements of free Ub concentrations as they change, for example, during enzymecatalyzed deubiquitination reactions.

With the dual goals of having a simple, reliable method to quantify cellular Ub pools and a sensitive and versatile real-time DUB assay, we embarked on development of sensors for free Ub detection and quantitation. To distinguish the free, activated, and conjugated Ub pools, we developed protocols to convert either thioesterified or (iso)peptideconjugated $\mathrm{Ub}$ into free $\mathrm{Ub}$ for subsequent quantitation with a sensor reagent. To engineer the sensors, our general strategy was to fuse genetically two or three Ub binding domains (UBDs) of known structure that bind to non-overlapping Ub surfaces, and to exploit avidity effects to achieve high affinity and selectivity. To convert the binding proteins into sensors, we attached fluorescent dyes whose intensity changed in response to binding by free Ub. We demonstrate that the sensors provide convenient means to (i) measure free, activated, and conjugated intracellular Ub; (ii) quantify deubiquitination of unlabeled (poly)Ub-protein conjugates in real-time DUB assays; and (iii) localize and quantify endogenous free Ub by fluorescence microscopy of fixed cells.

\section{Results}

\section{Design and characterization of the sensors}

The free Ub sensors were developed to have high affinity and selectivity for free Ub and report binding events via a strategically placed fluorophore. Our design strategy was to assemble Ub binding proteins from multiple UBDs linked in tandem; peptide linkers were kept to a minimum length in order to promote avidity while minimizing the entropic cost of binding. Most UBDs bind Ub through interactions with one of three different surfaces: the Ub hydrophobic patch surrounding residue 144, the Ub C-terminal tail, and the surface around D58 (Fig. 1a, upper panel). Individually, UBDs bind Ub with only modest affinity $\left(K_{d}=10^{-5}\right.$ to $\left.10^{-3} \mathrm{M}\right)$, but by linking two or three weak-binding UBDs that 
target distinct Ub surfaces, we expected that high affinity could be achieved overall. An early version of such an avidity-based binder that we call tIVR employed IsoT ${ }^{\text {Buz }}$, Vps27 ${ }^{\mathrm{UIM}}$, and Rabex $5^{\mathrm{Ruz}}$ domains fused in tandem with flexible peptide linkers (Fig. 1a, lower left panel and Supplementary Fig. S1). The IsoT ${ }^{\text {Buz }}$ domain $\left(K_{d}=3 \mu \mathrm{M} \text { for } U b\right)^{16}$, which binds primarily to residues at the Ub C-terminus, conferred selectivity for free $\mathrm{Ub}$, whereas the $\operatorname{Vps} 27^{\mathrm{UIM}}\left(\mathrm{K}_{d}=117 \mu \mathrm{M}\right)^{17}$ and Rabex ${ }^{\text {Ruz }}\left(\mathrm{K}_{\mathrm{d}}=12 \mu \mathrm{M}\right)^{18,19}$ domains worked synergistically with Iso $T^{\mathrm{Buz}}$ to increase overall affinity and specificity for $\mathrm{Ub}$.

To measure the affinity between tIVR and free $\mathrm{Ub}$, we first titrated a fluorescent $U b$ derivative (Atto532-Ub(S20C); see Methods) with tIVR and determined a $0.4 \mathrm{nM} \mathrm{K}$ based on an observed 3.5-fold fluorescence decrease upon formation of the 1:1 complex (Supplementary Fig. S2a). Then, by taking advantage of the high affinity and fluorescence intensity change of Atto532- $\mathrm{Ub}(\mathrm{S} 20 \mathrm{C})$ upon binding to tIVR, we determined tIVR affinities for $\mathrm{Ub}$ and other ligands by use of competition binding assays (Fig. 1b). We found that free $U b$ binds to tIVR with a $K_{d}$ of $19 \mathrm{nM}$ (measured as a $\mathrm{K}_{\mathrm{i}}$; Fig. $1 \mathrm{~b})$; the nearly 50 -fold stronger binding of Atto532- $\mathrm{Ub}(\mathrm{S} 20 \mathrm{C})$ indicates that the Atto532 dye contributes favorably $(\Delta G \sim-0.2 \mathrm{kcal} / \mathrm{mol})$ to the interaction with $\mathrm{tIVR}$. The competition assays additionally showed that tIVR has high selectivity against the Ub-like (UbL) protein Nedd8 and against Ub derivatives that lack a free C-terminal carboxylate (Fig. 1b). Among the many different UbL proteins found in eukaryotes ${ }^{20}$, Nedd8 is most similar to $\mathrm{Ub}$ in its sequence and tertiary structure, and its C-terminal four amino acids are identical ${ }^{21}$; nonetheless, tIVR has a 3000 -fold preference for Ub. A similarly large discrimination was observed against Ub-GB1, a mimic of a Ub-protein conjugate in which the Ub C-terminus is extended by the Protein G 56-amino acid B1 domain, and even addition of the small adduct hydrazine to the Ub C-terminus decreased tIVR affinity 150 -fold relative to free $\mathrm{Ub}$ (Fig. 1b).

In order to provide the sensor with a direct readout of $U b$ binding, we explored sitespecific labeling with fluorescent dyes (Supplementary Fig. S3). We found that tIVR modified with Atto532-maleimide on C130 showed a 3-fold fluorescence increase upon Ub binding (Supplementary Fig. S2b). By replacing the $\mathrm{Vps} 27^{\mathrm{UIM}}$ with the S5a UIM 
domain and introducing two amino acid substitutions in the IsoT ${ }^{\text {Buz }}$ domain (Fig. 1a and Supplementary Fig. S1), we developed a second-generation sensor, tISR, that binds to free Ub nearly 10-times tighter than tIVR (Supplementary Fig. S2c,d). Atto532-labeled tISR showed a 6-fold fluorescence increase upon binding of free Ub, but the fluorophore reduced affinity for Ub so that Atto532-labeled tISR and unlabeled tIVR showed similar $\mathrm{K}_{\mathrm{d}}$ values of $24.3 \mathrm{nM}$ and $25.4 \mathrm{nM}$, respectively (Fig. 1c,e and Supplementary Fig. S2b).

A third generation of free Ub sensor was developed with the goal of achieving even greater affinity. Ubiquilin-1 UBA (UQ1 ${ }^{\mathrm{UBA}}$ ) was used to replace the tIVR/tISR UIM because of its relatively tight binding to the Ub hydrophobic patch $\left(K_{d}=22 \mu M\right)^{22,23}$ and we predicted that a shorter linker ( 2 amino acids versus 5 with tIVR or tISR) could be used to connect the UQ1 ${ }^{\mathrm{UBA}} \mathrm{C}$-terminus with the $\mathrm{N}$-terminus of IsoT ${ }^{\mathrm{Buz}}$. The shorter linker was expected to reduce the entropic cost of complex formation. We conjugated Atto532 to UQ1 ${ }^{\text {UBA }}$ G573C in tUI (Fig. 1a, lower right panel); titration with free Ub revealed a 3-fold increase in fluorescence and a remarkably low $\mathrm{K}_{d}$ of 66 pM (Fig. 1d,e). In an independent experiment, we measured the association and dissociation rate constants for the Atto532-tUI-Ub complex from which we calculated a $\mathrm{K}_{d}$ of $56.4 \pm 1.2$ pM (Supplementary Fig. S4), which is in excellent agreement with the results from titrations done at equilibrium. To our knowledge, this very high affinity is unprecedented for binding to a single Ub. Atto532-tUI showed exceptional selectivity against Ub Cterminal conjugates, having a binding preference for free Ub over Ub-GB1 of $>10^{6}$. With respect to discrimination of Ub from UbL proteins, Atto532-tUl has 120-fold higher affinity for Ub than Nedd8. The substantially higher selection against Nedd8 seen with the tIVR and tISR sensors can be attributed to the Ruz domain, which is absent in tUI. Nonetheless, as described below, tUl's selectivity coupled with its exceptional affinity make it the first choice for most in vitro applications.

The results above demonstrated the high selectivity of sensors engineered to bind Ub that has a free C-terminus. In cells, multiple forms of Ub can fulfill that criterion; most notably, the proximal Ub in a "free" or "unanchored" polyUb chain would have its Cterminal amino acids available for binding by the sensor Buz domain. Inspection of the 
sites on Ub used for Ub-Ub linkages in polyUb (i.e., Ub's seven lysine $\varepsilon$-amines plus the $\alpha$-amine on M1) showed that they are not occluded when bound by the various UBDs used in the sensors (Fig. 1a). These models are consistent with studies that have shown little or no discrimination by individual UBA, UIM, or Buz domains for binding Ub in chains with different Ub-Ub linkages ${ }^{16,22,23}$. Accordingly, competition by $\mathrm{Ub}_{2}$ or $\mathrm{Ub}_{4}$ linked through $\mathrm{M} 1, \mathrm{~K} 6, \mathrm{~K} 29$, K33, K48, or $\mathrm{K} 63$ revealed little difference among them or from free monoUb in binding to tIVR (Fig. 1e,f). Although not tested directly, based on previous findings of linkage-independent binding of UBA domains ${ }^{22,23}$ and the small tUI footprint modeled onto Ub (Fig. 1a), we anticipate similar linkage-independent binding by tUI to unanchored polyUb.

Another modification to Ub that potentially could affect detection by the sensors is phosphorylation. Multiple serine and threonine phosphosites have been found on $\mathrm{Ub}^{24}$; as with Ub lysines, most of these do not show overlap with the surfaces bound by our sensor UBDs (Fig. 1a). Ub phosphorylation on S65, which has functions in Ub-mediated signaling and mitophagy in particular, has been the most intensively studied phosphoUb species $^{25-28}$. When we titrated Atto532-tUI with Ub(pS65) we determined a $\mathrm{K}_{d}$ of $262 \mathrm{pM}$ (Fig. 1d,e), indicating 4-fold weaker binding than with Ub. Wauer et al. have shown by NMR that Ub(pS65) exists in an equilibrium between two principal conformers ${ }^{29}$. The major form $(\sim 70 \%)$ is essentially like unmodified Ub, whereas in the minor conformer $(\sim 30 \%)$ movement of the $\beta 5$ strand retracts the normally-extended C-terminal residues into the body of Ub and displaces a key component of the hydrophobic patch. Because interactions with Buz and UBA domains are likely to be disrupted in the minor Ub(pS65) conformer, we expect that tUI would bind tightly only to the major conformer; thus, the $\mathrm{Ub}$ versus $\mathrm{Ub}(\mathrm{pS65}) \mathrm{K}_{d}$ difference could in part reflect the equilibrium where only a fraction of $\mathrm{Ub}(\mathrm{pS65})$ is in the binding-competent conformation.

\section{Real-time deubiquitination assays with label-free substrates}

Quantitative activity assays are essential to understand the regulations and specificities of DUBs. Typically physiological substrates of DUBs are not used in quantitative assays due to limited availability and lack of good methods to quantify products. Moreover, real- 
time monitoring of activity — the preferred approach to high-precision kinetics studies has been virtually impossible with physiological DUB substrates. For these reasons, artificial Ub derivatives such as Ub-(7-amido-4-methylcoumarin) or Ub-protein conjugates (e.g., diUb) with pairs of fluorophores that enable FRET-based assays are used $^{30,31}$. Our free Ub sensors now make it possible to develop less restrictive DUB assays that could employ virtually any Ub conjugate as a substrate. As an example, we used Atto532-tIVR to monitor DUB-catalyzed release of free Ub (or unanchored polyUb) in a continuous fluorometric assay. For a model substrate, we used K48-linked Ub5 conjugated to the $\mathrm{N}$-terminus of ovomucoid first domain (OM); to provide a second means to assay reactions, the OM moiety was modified with Lucifer Yellow dye (LY) to facilitate detection after SDS-PAGE ${ }^{32}$. For the DUB, we used human OTUB1, which selectively cleaves K48 Ub-Ub isopeptide linkages ${ }^{33}$ and is activated allosterically by interaction with certain E2 enzymes ${ }^{34}$. Without OTUB1, Ub 5 OM(LY) and Atto532-tIVR showed no fluorescence change, whereas enzyme addition initiated a fluorescence increase indicating release of free (poly)Ub (Fig. 2). Furthermore, in agreement with the report by Wiener et al. ${ }^{34}$, addition of $\mathrm{UbcH} 5 \mathrm{c}$ stimulated the deubiquitination activity. SDS-PAGE of samples from the reaction mixtures confirmed the results from the sensor (Supplementary Fig. S5). Because OTUB1 is selective for K48-linked polyUb, Ub ${ }_{1}^{-}$ $\mathrm{OM}(\mathrm{LY})$ accumulated upon OTUB1 digestion, even in the presence of UbcH5c (lane 6, Supplementary Fig. S5a-c). This remaining conjugated Ub could be cleaved by the nonspecific DUB Usp2cc (lane 7, Supplementary Fig. S5a-c). The release of free Ub determined in real-time using the sensor agreed with the amounts calculated by quantifying the LY-labeled gel bands (Supplementary Fig. S5d).

\section{Ub pool quantitation in cell lysates}

We next developed methods that use the sensors to quantify cellular Ub pools. Our goal was to generate a workflow for routine measurements that would not depend on expensive, time-intensive steps requiring chromatography and mass spectrometry to separate and detect different Ub populations. The general approach, in which the sensor (e.g., Atto532-tUI) fluorescence is measured with and without addition of cell lysate or other sample, promised to be simple and direct. The main challenge was to 
develop conditions to prevent appearance of free Ub due to disassembly of conjugates by endogenous DUBs or from spontaneous hydrolysis of Ub thioesters.

Our strategy was to lyse cells and quickly inactivate endogenous DUBs and other proteases (see Methods) and then treat each sample in three ways to differentially convert Ub pools to the free-Ub form for measurement with the sensor (Fig. 3a). In order to measure endogenous free Ub without interference from chemically-labile Ub thioesters, samples were treated with hydrazine to rapidly and selectively convert all Ub thioesters into Ub C-terminal hydrazide; Ub-hydrazide is stable and, relative to free Ub, gives a negligible response with the sensor (Fig. 1b-e and Supplementary Fig. S6). Thus, sensor fluorescence of a hydrazine-treated sample will measure endogenous free Ub. With a second portion of the sample, $\beta$-mercaptoethanol was used to release Ub from Ub thioesters; measurement with the sensor then will report the sum of the endogenous free and thioester Ub pools ${ }^{35}$. A third portion was incubated with Usp2cc, a truncated DUB that can deubiquitinate virtually all forms of conjugated $\mathrm{Ub}^{36}$; when used in combination with a thiol reducing agent, all forms of $\mathrm{Ub}$ in the sample are converted to free Ub and the sensor readout will report the total Ub. By deducting the sum of the activated and free $\mathrm{Ub}$ from the total Ub, we can determine the amount of Ub that had been in (poly)Ub-proteins or other conjugates.

These assays were first used to quantify the amounts of free, activated, and conjugated Ub in HeLa cells (Fig. 3); the results are in good agreement with other reports ${ }^{15,37,38}$. The sensor assays then were used with HeLa cells after treatment with inhibitors of the E1 Ub-activating enzyme or proteasome (Fig. 3b,c). As expected ${ }^{39}$, the E1 inhibitor Compound 1 (C1) dramatically increased free Ub with a concomitant loss of activated $\mathrm{Ub}$ and most Ub-protein conjugates (Fig. 3b). Conversely, proteasome inhibition by bortezomib (BTZ) promoted accumulation of Ub conjugates that reached a maximum at $1 \mathrm{~h}$ and then persisted through a $4 \mathrm{~h}$ treatment (Fig. 3c; Supplementary Fig. S7). The conjugate increase was accompanied by a modest depletion of activated Ub and a twofold decrease in free Ub, presumably due to impaired proteasome-dependent recycling of $\mathrm{Ub}$ from conjugates. Different from this result, proteasome-inhibited MEF cells 
exhibited little change in free Ub levels, even though conjugated Ub increased 50\%; instead, the total amount of $\mathrm{Ub}$ increased, likely due to increased expression of $\mathrm{Ub}$ genes $^{40}$.

\section{Quantitation of endogenous free Ub in fixed cells}

We realized that Atto532-tUI, with its high affinity and specificity for free Ub and conjugated fluorophore, could be an ideal tool to localize endogenous, intracellular free Ub. Initially, fixed cells were stained directly with Atto532-tUI, but high background fluorescence, most likely from nonspecific binding by the fluorophore, reduced sensitivity (data not shown). Therefore, we used instead a hemagglutinin-tagged version of tUI (HA-tUI) followed by detection with an anti-HA antibody. We confirmed the specificity of HA-tUI for free Ub by performing control experiments where fixed cells were incubated with HA-tUI together with excess free Ub. The fluorescence observed in these competition experiments was negligible, suggesting that the staining with HA-tUI is specific for cellular Ub (Supplementary Fig. S8). A diffuse intracellular distribution of free Ub was expected based on its small size (8.6 kDa) and negligible self-association ${ }^{41}$. Staining with HA-tUI was observed throughout the cytoplasm and nucleus in HeLa, U2OS, MEF and RPE1 cell lines (Fig. 4a). Compared to K48-linked chains and monoand polyubiquitylated proteins, free Ub staining is evenly distributed through the whole cell (Supplementary Fig. S9). GFP-Ub that had been mutated to prevent its conjugation to other proteins was similarly diffuse when expressed in mammalian cells ${ }^{13}$.

Staining with HA-tUI offers an alternative to solution-based assays to monitor changes in free Ub during growth or in response to different stresses. After proteasome inhibition by incubation with BTZ for $1 \mathrm{~h}$, HeLa, U2OS, MEF and RPE1 cells showed decreased staining with HA-tUI based on their 1.3 to 1.5-fold lower anti-HA antibody fluorescence relative to control (i.e., vehicle only) cells. As expected, E1 inhibition increased the free Ub (2-fold in MEF and RPE cells, and 3-fold in HeLa and U2OS cells) (Fig. 4b,c). Although proteasome inhibition decreased free Ub staining in all four cell lines, the intracellular distributions of free Ub appeared unaffected. For RPE1 cells, ratios of cytoplasmic to nuclear staining were not changed significantly by incubation with BTZ (control cells, $0.85 \pm 0.07, n=5$; BTZ, $0.89 \pm 0.08, n=4$ ). In contrast to the results with 
BTZ, increased staining was observed after E1 inhibition and cell-to-cell variability was greater overall (Fig. 4a-c). The average changes in staining of HeLa cells are consistent with the in-solution assays, whereas for MEF cells the staining showed $\sim 25 \%$ less free $\mathrm{Ub}$ after proteasome inhibition than was determined from the in-solution assays (Figs. $3 c$ and $4 a-c)$. Possibly, because hydrazine treatment was not used with the imaged cells, some of the free $\mathrm{Ub}$ detected by staining could have originated from spontaneous hydrolysis of the Ub-thioester pool, thereby inflating the "free" Ub and confounding direct comparison of the two assays. The staining with $\mathrm{tUI}$ also revealed cell-cycle dependent differences in free Ub levels. RPE1 cells undergoing mitosis showed a 1.6fold increase in tUl staining compared with interphase cells (Fig. 4d). The increase in free $\mathrm{Ub}$ may be due, at least in part, to the large-scale deubiquitination of $\mathrm{H} 2 \mathrm{~A}$ histones observed for mitotic cells ${ }^{42}$.

\section{Discussion}

Individually, most UBDs have only modest affinity for Ub and are typically found together with other binding domains (and sometimes additional UBDs) to promote binding to specific types of polyUb or Ub-protein conjugates. From genetic fusions of multiple UBDs, we have engineered new proteins with specificity and extremely high affinity for monomeric free $\mathrm{Ub}$. Our basic strategy was to use the Buz domain to direct binding to $\mathrm{Ub}$ with an unconjugated $\mathrm{C}$-terminus, and to increase affinity with one or two additional UBDs that interact with Ub on non-overlapping surfaces. A critical aspect of the design strategy was to maximize the effect from avidity. This was achieved by having multiple UBDs bind simultaneously and by minimizing the entropy lost upon complex formation through careful selection of peptides linking the UBDs. Avid binding can boost affinity by combining the contributions of individual binding domains in a complex assembled from a multivalent ligand and a corresponding multivalent binder. The Gibbs free energy for binding overall $\left(\Delta G_{\text {total }}\right)$ can be approximated as the sum of the individual binding domain (BD) interaction free energies $\left(\Delta G_{B D 1}, \Delta G_{B D 2}\right.$, etc.) plus the unfavorable free energy due to reduction in entropy (predominantly, losses in translational and rotational entropy) from having all the binding domains linked together $\left(\Delta G_{S}\right)^{43}$ : 


$$
\Delta \mathrm{G}_{\text {total }}=\Delta \mathrm{G}_{\mathrm{BD} 1}+\Delta \mathrm{G}_{\mathrm{BD} 2} \cdots+\Delta \mathrm{G}_{\mathrm{BDn}}+\Delta \mathrm{G}_{\mathrm{S}}
$$

To achieve "perfect" avid binding and maximize affinity, $\Delta \mathrm{G}_{\mathrm{S}}$ should be close to zero. For Ub binding by a tandem-UBD (tUBD) protein such as tIVR, we can write:

$$
\Delta \mathrm{G}_{\mathrm{TUBD}}=\Delta \mathrm{G}_{\mathrm{UBD} 1}+\Delta \mathrm{G}_{\mathrm{UBD} 2}+\Delta \mathrm{G}_{\mathrm{UBD} 3}+\Delta \mathrm{G}_{\mathrm{S}}
$$

$\Delta G_{U B D}$ can be calculated for each UBD based on the $K_{d}$ values reported for Ub binding by the individual IsoT ${ }^{\mathrm{Buz}}, \mathrm{Vps} 27^{\mathrm{UIM}}$, and Rabex $5^{\mathrm{Ruz}}$ domains (i.e., -7.53, -5.25, and -6.62 $\mathrm{kcal} \cdot \mathrm{mol}^{-1}$, respectively); similarly, $\Delta \mathrm{G}_{\mathrm{tIVR}}=-10.52 \mathrm{kcal} \cdot \mathrm{mol}^{-1}$ can be calculated from the tIVR-Ub $K_{d}$ of $19.3 \mathrm{nM}$ (Fig. 1e). Thus, although avidity promotes tight binding by tIVR, it's at the cost of $\Delta G_{S}$, which is $(-10.52)-(-19.40)=8.88 \mathrm{kcal} \cdot \mathrm{mol}^{-1}$. This substantial penalty reduced tIVR affinity by $>10^{6}$ from a theoretical $\mathrm{K}_{d}$ of $5.9 \times 10^{-15} \mathrm{M}$.

Remarkably, tUl's affinity for Ub (K $=194$ pM; Supplementary Fig. S10) is only 3-times weaker than what would be predicted for perfect avid binding by the combination of the UQ1 ${ }^{\text {UBA }}$ domain $\left(\mathrm{K}_{d}=22 \mu \mathrm{M}\right)^{22,23}$ and the Buz domain $\left(\mathrm{K}_{d}=3 \mu \mathrm{M}\right)^{16}$. Contributing to the much greater efficiency of avidity seen with tUI relative to tIVR is that tUI has only one linker peptide (versus two in tIVR), and its linker is very short (Supplementary Fig. S1). Thus, a likely route to increase affinity for tIVR or tISR is further optimization of the interdomain linkers. On the other hand, a large increase in affinity by tUI would have to come from tighter-binding versions of one or both of its UBDs, as the linker peptide already is nearly optimum.

There are several ways in which our tUBD binders for free Ub could be improved in affinity or for specific applications. Affinity might be increased, as noted above, with either modified linkers or alternative UBDs. Our designs have utilized only a few of more than 20 types of UBDs ${ }^{44,45}$; moreover, other $\mathrm{Ub}$ binding proteins such as catalyticallyinactive DUBs might be used as components of tUBD-type fusion constructs ${ }^{46}$. Particularly intriguing is the prospect of tailoring recognition by incorporation of a UBD that would select either for or against specific modifications on the Ub. For example, although tIVR binds equally well to free polyUb chains of different Ub-Ub linkage types (Fig. 1f), alternatives to the Ruz or UIM domain could be developed in which steric clash prevents binding to Ub conjugated at particular lysine(s). Similarly, UBD (or linker) 
modifications might be made to introduce selectivity for phosphoUb derivatives. In addition, alternative fluorophores and attachment sites can be explored further to improve sensor sensitivity and dynamic range.

As multiple DUBs have been implicated in human disease ${ }^{47}$, the sensors have potential to facilitate drug development. Although some DUB inhibitors have been tested clinically ${ }^{47}$, inhibitor development remains limited by difficulties in establishing highthroughput screens that can employ physiological DUB substrates. Important innovations of the sensor-based real-time assays are that virtually any Ub conjugate can be used as the substrate, and it is unnecessary to have the substrate labeled.

Other likely applications of the free Ub sensors are quantitations of Ub pools using the protocols we have described for in-solution assays or cell staining. Many neurological disorders disrupt Ub homeostasis and show aggregates of ubiquitinated proteins or a general depletion of free Ub. Staining by tUl offers a unique approach to examine, for example, neurons at the single-cell level to understand better the effects of genetic or environmental perturbations on Ub homeostasis and intracellular distribution. Additionally, extracellular free Ub has been suggested as a biomarker for trauma and disease $^{48}$; here, the free Ub sensors can replace the less specific antibody-based assays typically used to quantify extracellular Ub. Ultimately, we envision that the free Ub binders and the fluorescent sensors developed from them will provide effective tools to capture, deplete, quantify, or visualize free Ub in vitro and in cells. 


\section{Methods}

\section{Materials and protein preparation}

tIVR, tISR, tUI, Ub-GB1, and UbcH5c were cloned into pET28a and transformed into BL21-CodonPlus (DE3) E. coli cells for protein expression. Expression was induced by the addition of $0.4 \mathrm{mM}$ IPTG to cells grown at $37{ }^{\circ} \mathrm{C}$ to $\mathrm{OD}_{660 \mathrm{~nm}}=0.6-0.8$, and then growth was continued at $25{ }^{\circ} \mathrm{C}$ for $8 \mathrm{~h}$. The cells were harvested by centrifugation at $3,200 \times g$, resuspended in ice-cold Buffer $\mathrm{A}$ ( $20 \mathrm{mM}$ sodium phosphate, $\mathrm{pH} 7.4,500 \mathrm{mM}$ $\mathrm{NaCl}, 10 \mathrm{mM}$ imidazole, and $10 \mathrm{mM} \beta$-mercaptoethanol), and lysed by sonication; the lysates were clarified by centrifugation for $30 \mathrm{~min}$ at $4{ }^{\circ} \mathrm{C}$ at $20,199 \times \mathrm{g}$. A Histrap HP column (GE Healthcare, 17-5248-02) was used to purify the proteins from the lysates. Samples were applied to the column equilibrated with Buffer $A$, and after washing with 20 column volumes, bound proteins were eluted with a linear gradient to $500 \mathrm{mM}$ imidazole in Buffer A. The proteins were further purified by gel filtration through a Superdex 75 column (GE Healthcare, 29-1487-21) eluted with pH 7.4 PBS and $1 \mathrm{mM}$ DTT or $1 \mathrm{mM}$ TCEP. Purity was confirmed by SDS-PAGE. Ub ${ }^{49}$, Nedd $8^{50}$, Usp2cc ${ }^{36}$, and $\mathrm{Ub}_{5}-\mathrm{OM}(\mathrm{LY})^{32}$ were prepared as described. OTUB1 was a gift from $\mathrm{C}$. Wolberger (Johns Hopkins University). K6, K11, K27, K29, K33, K48, and K63-linked Ub ${ }_{2}$ chains were purchased from UbiQ Bio (Amsterdam), Ub(pS65) was from BostonBiochem (Cambridge, MA), and M1-linked $\mathrm{Ub}_{4}$ was prepared as described ${ }^{51}$.

\section{Synthesis of Ub-hydrazide}

$\mathrm{Ub}(1.5 \mathrm{mM}$ ) was incubated with $10 \mathrm{mM} \mathrm{ATP}, 10 \mathrm{mM} \mathrm{MgCl}, 100 \mathrm{mM}$ sodium 2mercaptoethanesulfonate (MESNA; Fluka), and $100 \mathrm{nM}$ mouse E1 in $20 \mathrm{mM}$ HEPES ( $\mathrm{pH} \mathrm{8.0)} \mathrm{for} 3 \mathrm{~h}$ at $37^{\circ} \mathrm{C}$ to form Ub-MESNA thioester (confirmed by mass spectrometry; see Supplementary Fig. S6b). The Ub-MESNA then was incubated in $300 \mathrm{mM}$ aqueous hydrazine for $30 \mathrm{~min}$ at $37{ }^{\circ} \mathrm{C}$ to form Ub-hydrazide. The reaction product was diluted 25-fold with $50 \mathrm{mM}$ ammonium acetate, adjusted to $\mathrm{pH} 4.5$ (Buffer B), and purified by cation-exchange chromatography on a Mono S column (GE Healthcare, 17-0547-01). The column was washed with 20 volumes of Buffer B and eluted with a linear gradient of $0-1 \mathrm{M} \mathrm{NaCl}$ in the same buffer. The purified Ub-hydrazide was confirmed by mass spectrometry (Supplementary Fig. S6b). 


\section{Fluorophore labeling}

Sensor proteins were labeled at cysteine with fluorophore-maleimide dyes from ATTOTEC GmbH (Atto dyes; see Supplementary Fig. S3), Molecular Probes (Alexa Fluor 488 ), or Anaspec (fluorescein). Fluorophore-maleimide dyes (1.5 to 5 -fold molar excess) were incubated with $50 \mu \mathrm{M}$ sensor in $50 \mathrm{mM}$ HEPES, $\mathrm{pH} 7.4,100 \mathrm{mM} \mathrm{NaCl}$ for $2 \mathrm{~h}$ at $25^{\circ} \mathrm{C}$. Excess dyes were quenched by incubation with $10 \mathrm{mM} \beta$-mercaptoethanol for 10 min at $25{ }^{\circ} \mathrm{C}$. To remove excess dyes, the reaction product was bound to Ni-NTA resin (Thermo Fisher) equilibrated with Buffer $A$, the resin was washed 5 times with the Histrap binding buffer, and sensor proteins were eluted with Histrap elution buffer. Labeling was confirmed by SDS-PAGE and then scanning the gel for fluorescence using a Typhoon FLA 9500 (GE Healthcare Life Sciences). Degree of labeling (DOL) and concentrations of the labeled proteins were calculated by the equations below.

$$
\begin{aligned}
& \mathrm{DOL}=\frac{\mathrm{A}_{\mathrm{m}} \times \varepsilon_{\text {prot }}}{\left(\mathrm{A}_{280}-\mathrm{A}_{\mathrm{m}} \times \mathrm{CF}_{280}\right) \times \varepsilon_{\mathrm{m}}} \quad \mathrm{CF}_{280}=\frac{\varepsilon_{280}}{\varepsilon_{\mathrm{m}}} \\
& \text { Protein concentration }(\mathrm{M})=\frac{\left(\mathrm{A}_{280}-\left(\mathrm{A}_{\mathrm{m}} \times \mathrm{CF}_{280}\right)\right)}{\varepsilon_{\text {prot }}}
\end{aligned}
$$

In the equations, $A_{m}$ represents the absorbance at the dye absorption maximum, $A_{280}$ is absorbance at $280 \mathrm{~nm}$ of the labeled protein, $\varepsilon_{\text {prot }}$ is the extinction coefficients at $280 \mathrm{~nm}$ of the protein, $\varepsilon_{280}$ is the extinction coefficient at $280 \mathrm{~nm}$ of the dye alone, $\varepsilon_{\mathrm{m}}$ is the extinction coefficient at the absorption maximum of the dye, and $\mathrm{CF}_{280}$ is the correction factor at $280 \mathrm{~nm}$.

\section{Binding assays}

All binding assays were done in PBS buffer, $\mathrm{pH} 7.4$, supplemented with $0.05 \%$ Brij35 and either $0.2 \mathrm{mg} / \mathrm{ml}$ ovalbumin or GB1 protein, and either $1 \mathrm{mM}$ DTT or $1 \mathrm{mM} \mathrm{TCEP.} \mathrm{A}$ FluoroMax-4 spectrofluorimeter (HORIBA Scientific) was used to measure fluorescence intensity in the binding assays. $K_{d}$ and $K_{i}$ values were calculated by fitting with a singlesite binding model ${ }^{52}$ using Prism 6 (GraphPad Software). Because Atto532-tUl has exceptionally high affinity for $\mathrm{Ub}, 10 \mathrm{pM}$ Atto532-tUI was used in the binding assays to keep its concentration below the $\mathrm{K}_{d}$ for $\mathrm{Ub}$. To improve detection of the fluorescence 
from this low concentration of Atto532-tUl, we increased the assay volume to $2.7 \mathrm{ml}$. The stock Ub titrated into the solution was $\leq 1.5 \%$ of the total volume.

\section{Stopped flow kinetics}

To determine the $\mathrm{k}_{\mathrm{off}}$ and $\mathrm{k}_{\mathrm{on}}$ rates of Atto532-tUI with free Ub, rapid kinetics were monitored by fluorescence using a MOS-500 spectrometer equipped with a SFM-4000 mixer (Bio-Logic Science Instruments) maintained at $25^{\circ} \mathrm{C}$. The excitation wavelength was set to $530 \mathrm{~nm}$ with a $5 \mathrm{~nm}$ bandwidth, and the fluorescence emission was detected with a $540-620 \mathrm{~nm}$ bandpass filter.

\section{Real-time DUB assays}

A Fluoromax-4 spectrofluorimeter (HORIBA Scientific) was used to monitor fluorescence of samples $(45 \mu \mathrm{L})$ in ultramicro quartz cuvettes (Hellma) at $25{ }^{\circ} \mathrm{C}$. The buffer was PBS, pH 7.4, with 0.05\% Brij35, $0.2 \mathrm{mg} / \mathrm{ml}$ ovalbumin, and $1 \mathrm{mM}$ DTT. A standard curve was generated to convert change in sensor fluorescence to the corresponding free Ub concentrations; e.g., 2 nM Atto532-tIVR was titrated with 2, 3, 6, 10, 18, and $32 \mathrm{nM} \mathrm{Ub}$, and the binding curve was fit as described above. Fluorescent intensities from real-time DUB assays then were converted to free Ub concentrations using the fitted binding equation. For deubiquitination of $\mathrm{Ub}_{5}-\mathrm{OM}(L Y)$, results were confirmed by SDS-PAGE of samples from the reaction mixtures and fluorescence imaging of the gel with a Typhoon FLA 9500 laser scanner (GE Healthcare Life Sciences).

\section{In-solution Ub pool assays}

Sample preparation. Cells were lysed in 100 mM MOPS, pH 6.0, 8 M urea, 20 mM NEM, and EDTA-free Complete protease inhibitors (Roche) by sonication and then centrifuged at $15,800 \times \mathrm{g}$. Total protein in the clarified extract was measured using the bicinchoninic acid (BCA) assay before being divided into three fractions for treatment with Usp2cc, $\beta$-mercaptoethanol, or hydrazine. The fraction to be treated with Usp2cc was diluted with digestion buffer (25 mM HEPES, pH 7.5, $140 \mathrm{mM} \mathrm{NaCl}$, and $10 \mathrm{mM}$ DTT) to reduce the urea to less than $2 \mathrm{M}$; to this, Usp2cc was added at a 1:10 
(Usp2cc:total protein) ratio and incubated at $37^{\circ} \mathrm{C}$ for $1 \mathrm{~h}$. To another fraction of the extract, $100 \mathrm{mM}$ CHES, pH 9, containing $150 \mathrm{mM} \beta$-mercaptoethanol was added and incubated at $37^{\circ} \mathrm{C}$ for $1 \mathrm{~h}$. The third fraction was incubated at $37^{\circ} \mathrm{C}$ for $1 \mathrm{~h}$ with freshlymade $200 \mathrm{mM}$ hydrazine- $\mathrm{HCl}, \mathrm{pH}$ 8.5. These samples were then diluted using PBS and $0.2 \mathrm{mg} / \mathrm{ml}$ ovalbumin to insure that [Ub] was within the linear range of the assay (e.g., with Att532-tUI, from 2-60 nM). Dilution was also performed to reduce the concentrations of urea $(<0.2 \mathrm{M})$, $\beta$-mercaptoethanol $(<20 \mathrm{mM})$, hydrazine $(<20 \mathrm{mM})$, which otherwise can interfere with binding by the sensor.

In-solution high-throughput assay to measure free Ub. Microplates (384-well SensoPlate Plus, Greiner Bio-One 781856) were used to measure free Ub concentrations of unknown samples in a high-throughput format. The plates were passivated by sequential treatment with $1 \%$ Hellmanex detergent, $1 \mathrm{M} \mathrm{KOH}$, and then $2 \%$ 1,7-dichloro-octamethyltetrasiloxane (Sigma-Aldrich) diluted in heptane, where each step was a 30 min soak followed by extensive washes with distilled water and finally airdrying. Typically, $24 \mu$ of a master mix containing $50 \mathrm{nM}$ Atto532-tUI and assay buffer (200 mM sodium phosphate, pH 7.5, $50 \mathrm{mM} \mathrm{NaCl,} 2 \mathrm{mM}$ DTT, 0.05\% Brij35, and 0.2 $\mathrm{mg} / \mathrm{ml}$ ovalbumin) was added to wells of the passivated 384-well plate. Then, to one set of wells, Ub standards were added and the remaining wells were used for samples (6 $\mu \mathrm{l})$. Fluorescence intensities were quantified with a Typhoon FLA 9500 laser scanner (GE Healthcare). The Ub concentrations of unknown samples were determined by interpolating the fluorescent signals on standard curves generated by titration with a standard of free Ub.

\section{Sample preparation for microscopy}

HeLa, U2OS, and MEF cells were cultured in DMEM (Gibco) supplemented with 10\% (v/v) FBS and $100 \mathrm{U} / \mathrm{mL}$ penicillin and $100 \mathrm{ug} / \mathrm{mL}$ streptomycin (Hyclone). RPE1 cells were cultured in DMEM/Ham's F-12, 50/50 mix (Corning). Cells were incubated for 1 hour with $1 \mu \mathrm{M}$ bortezomib (Ubiquitin-Proteasome Biotechnologies), $10 \mu \mathrm{M} E 1$ inhibitor (Compound 1; provided by Takeda Oncology, Cambridge, MA) or vehicle alone $(0.1 \%$ DMSO). Each experiment was performed a minimum of two times. 
We fixed cells at $<80 \%$ confluence with $4 \%$ paraformaldehyde in PBS for 30 min at $37^{\circ} \mathrm{C}$, permeabilized them with $0.1 \%$ Triton $\mathrm{X}-100$ for $10 \mathrm{~min}$ at room temperature and blocked for 1 hour with 5\% BSA and 0.5\% Tween 20 in PBS. Cells were stained for free Ub with $100 \mathrm{nM}$ tUl-HA diluted in blocking solution for $30 \mathrm{~min}$ at room temperature. As a negative control, the sensor was pre-incubated for $5 \mathrm{~min}$ at room temperature with 100 $\mu \mathrm{M} \mathrm{Ub}$ in blocking solution before addition to the samples. Next, cells were incubated overnight at $4{ }^{\circ} \mathrm{C}$ with anti-HA antibody (Sigma-Aldrich clone HA-7 or Bethyl Laboratories A190-108A; 1:1000 dilution), stained with Alexa Fluor 568-conjugated goat anti-mouse IgG (Thermo Fisher; 1:500 dilution), and mounted on slides using ProLong Diamond Antifade medium (Thermo Fisher). Some coverslips were also stained with anti-Ub (clone FK2, Biomol; 1:1,000 dilution) or anti-K48Ub (clone Apu2, Millipore; 1:200 dilution) primary antibodies and, subsequently, with Alexa Fluor 568-conjugated goat anti-mouse and Alexa Fluor 488-conjugated goat anti-rabbit (Thermo Fisher; 1:400 dilution) secondary antibodies. The HCS CellMask dye (Thermo Fisher) was added to the cells for half hour as a marker of cell boundaries for high-content fluorescence intensity-based measurements.

\section{Microscopy and image analysis}

Cells were imaged using a Zeiss LSM 880 confocal microscope with C-Apochromat 40X/1.20 W or Plan-Apochromat 63x/1.40 Oil DIC M27 objectives. Z-stack images were acquired with ZEN Black software (Version 14.0.9.201) at 0.746 $\mu \mathrm{m}$ intervals. ImageJ $1.51 \mathrm{~h}(\mathrm{NIH})$ was used to perform maximum intensity projections of z-sections and to calculate cell mean fluorescence intensity values; cell contours were drawn using HCS CellMask dye as a reference, and nuclei were identified with DAPI stain. Autofluorescence intensities recorded from unstained cells were subtracted from the tUl-fluorescence.

Three-dimensional reconstructions of RPE-1 cells were obtained with Imaris software (version 9.1.1, Bitplane AG) from serial z-sections acquired at $0.242 \mu \mathrm{m}$ increments. The Imaris surface creation tool was used to generate volume renderings and to quantify $\mathrm{tUI}$ fluorescence intensities in both interphase and mitotic cells. 


\section{Statistical Analysis}

Statistical calculations were performed with GraphPad Prism software and are described in the relevant figure legends. $P$ values less than 0.05 were considered significant. 
Figure 1

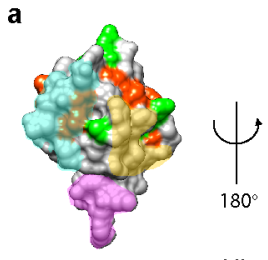

Ub

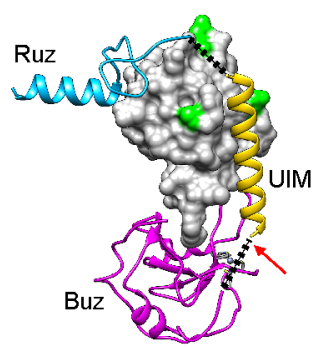

tIVR (IsoT ${ }^{\mathrm{Buz}}-\mathrm{V} p \mathrm{2} 27^{\mathrm{UM}}-\mathrm{Rabex}^{\mathrm{Ruz}}$ )

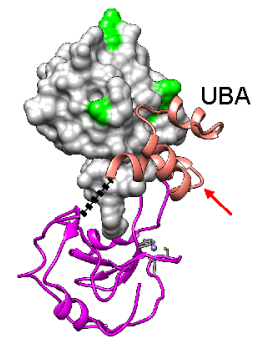

tUI (UQ1 $1^{\mathrm{UBA}}$-IsoT ${ }^{\mathrm{BU}}$ )

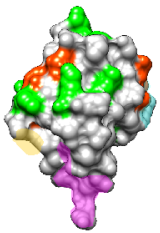

$$
\text { . }
$$

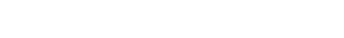

\begin{tabular}{cccccc} 
e & & & & & \\
Sensor & Ub & Nedd8 & Ub-GB1 & Ub-hydrazide Ub(pS65) \\
\hline tIVR & 19.3 & 57381 & 60309 & 3888 & - \\
$\mathrm{K}_{(}(\mathrm{nM})$ & \pm 1.7 & \pm 9486 & \pm 4335 & \pm 346 & - \\
\hline Atto532-tISR & 24.3 & 7338 & & 1042 & \\
$\mathrm{~K}_{\mathrm{d}}(\mathrm{nM})$ & \pm 5.3 & \pm 1529 & $>10^{5}$ & \pm 270 & - \\
\hline Atto532-tUI & 0.066 & 7.74 & & \\
$\mathrm{~K}_{\mathrm{d}}(\mathrm{nM})$ & \pm 0.016 & \pm 0.63 & & & - \\
\hline
\end{tabular}

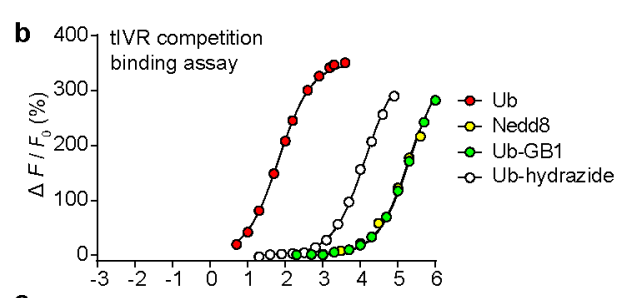

c $\left.{ }^{600}\right]$ Atto532-tISR direct
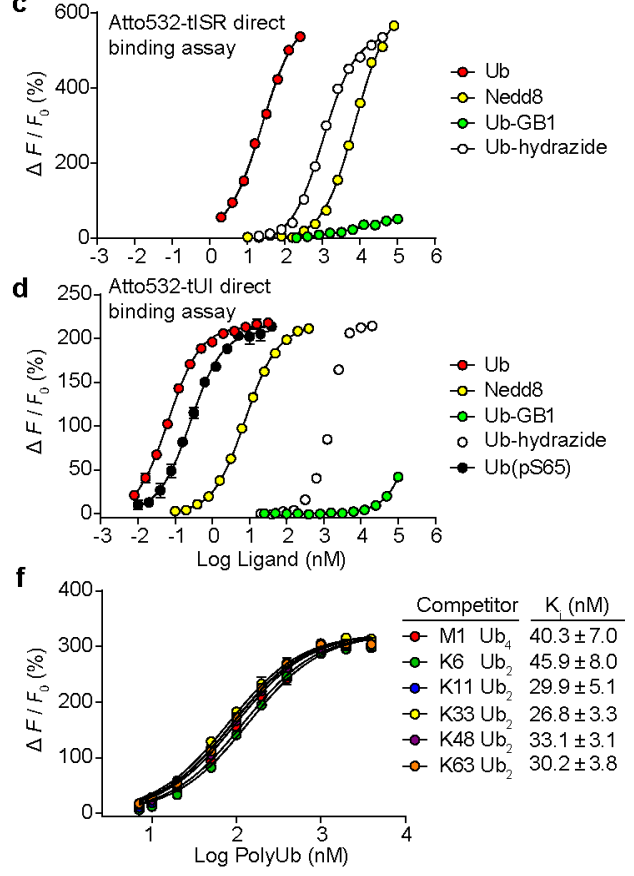

Fig. 1. Sensor design and characterization. a, Ub (upper panel) has distinct surfaces recognized by three classes of UBDs. Ub and UBDs are shown in surface and ribbon representations, respectively. For Ub (upper models), surfaces where Buz, UIM or UBA, and Ruz domains bind are in magenta, yellow, and cyan, respectively. Lysine and M1 sidechains (green) and phosphorylation sites (orange) are highlighted. Ub complexes with tIVR (lower left) and tUI (lower right) were modeled using individual UBD-Ub complex structures (PDB 2G45, 2FIF, 1Q0W, and 2JY6). The black dotted lines indicate linkers installed to connect UBDs, and red arrows show sites of fluorophore attachment. b, tIVR affinities for Ub and UbL derivatives were measured by competition with $1 \mathrm{nM}$ Atto532- $\mathrm{Ub}(\mathrm{S} 20 \mathrm{C})$ in the presence of $6 \mathrm{nM}$ tIVR. $\Delta F$ is the fluorescence intensity change of Atto532-Ub(S20C) upon addition of competitor and $F_{0}$ is the fluorescence without competitor. Fluorescence intensity changes of (c) Atto532-tISR or (d) Atto532tUI were measured by direct titrations with the Ub or UbL derivatives indicated and fit 
with a 1:1 binding model as described in Methods. e, Affinities $\left(K_{d}\right.$ or $\left.K_{i}\right)$ of the three sensors determined for the indicated Ub and UbL ligands. $\mathbf{f}$, Effects of Ub-Ub linkage type were assessed from competition binding assays with $0.8 \mathrm{nM}$ Atto532-Ub(S20C) and $6.0 \mathrm{nM}$ tIVR titrated with 7 to $4000 \mathrm{nM}$ of the indicated polyUb ligands. Statistical errors listed are standard deviations from the fits. Note that in $\mathbf{d}$, the titration with Ubhydrazide shows a saturation binding curve that might be due to trace contamination by free $\mathrm{Ub}$; therefore, a $\mathrm{K}_{d}$ value is not shown. Small error bars in $\mathbf{d}$ and $\mathbf{f}$, determined from duplicate assays, are masked by the point symbols. 
Figure 2
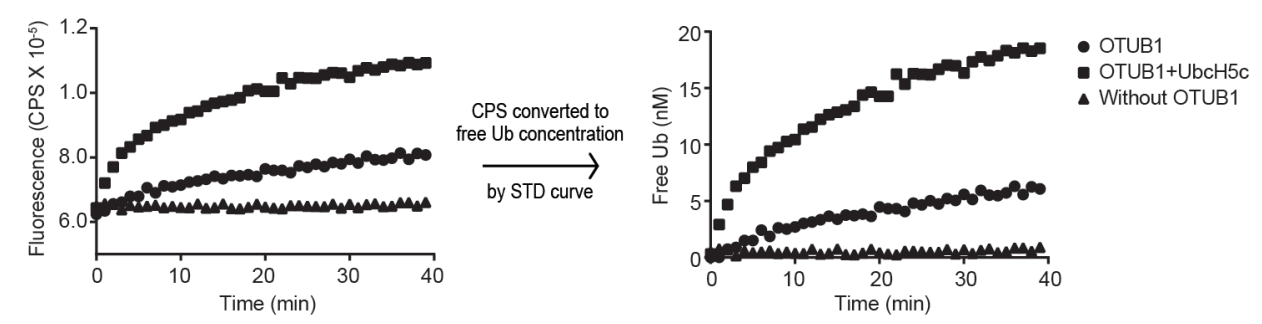

Fig. 2. Quantitative, real-time DUB activity assays with a Ub sensor. K48-linked $\mathrm{Ub}_{5} \mathrm{OM}(\mathrm{LY})(10 \mathrm{nM})$, a mimic of a polyubiquitinated protein conjugate, was mixed with 5 $\mu \mathrm{M}$ OTUB1 with or without $20 \mu \mathrm{M} \mathrm{UbcH5c}$ at $25^{\circ} \mathrm{C}$ in the presence of $2 \mathrm{nM}$ Atto532tIVR, and Atto532-tIVR fluorescence was monitored (left panel). A standard (STD) curve of Atto532-tIVR titrated with Ub (see Supplementary Fig. S5e) was used to convert the fluorescence intensity of Atto532-tIVR to free Ub concentration (right panel). 
Figure 3
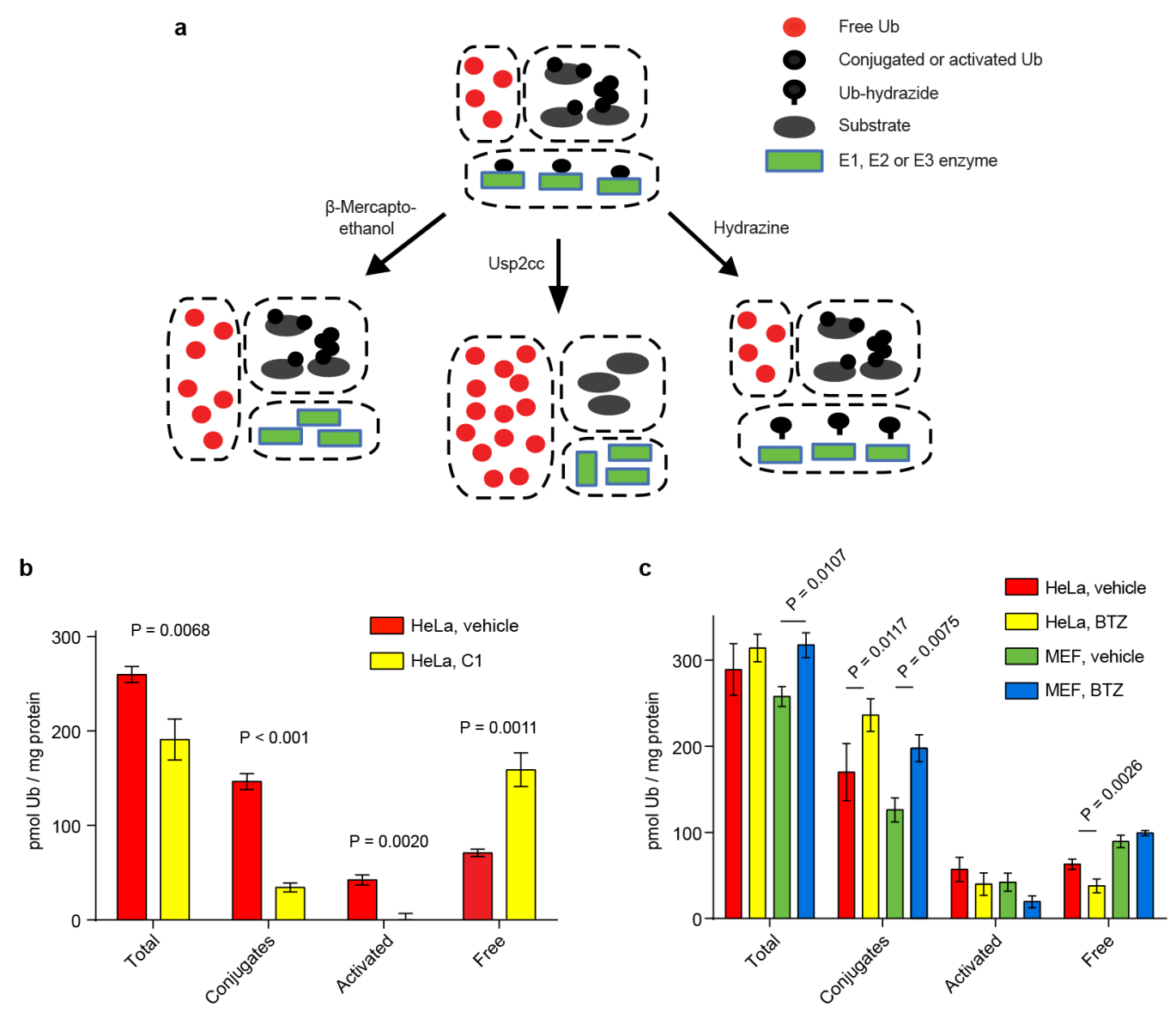

Fig. 3. Effects of cellular stresses on Ub pools. a, Scheme used for the in-solution Ub pool measurements. b,c, Quantitation of Ub pools in lysates of indicated cell lines after treatment with vehicle (DMSO) or (b) E1 inhibitor, $\mathrm{C} 1$, at $10 \mu \mathrm{M}$ or (c) proteasome inhibitor, BTZ, at $1 \mu \mathrm{M}$ for $1 \mathrm{~h}$. Statistical analyses by t-test (b) and ONE-WAY ANOVA with Bonferroni's adjustment (c); error bars represent \pm s.d. $(n=3)$. 
Figure 4

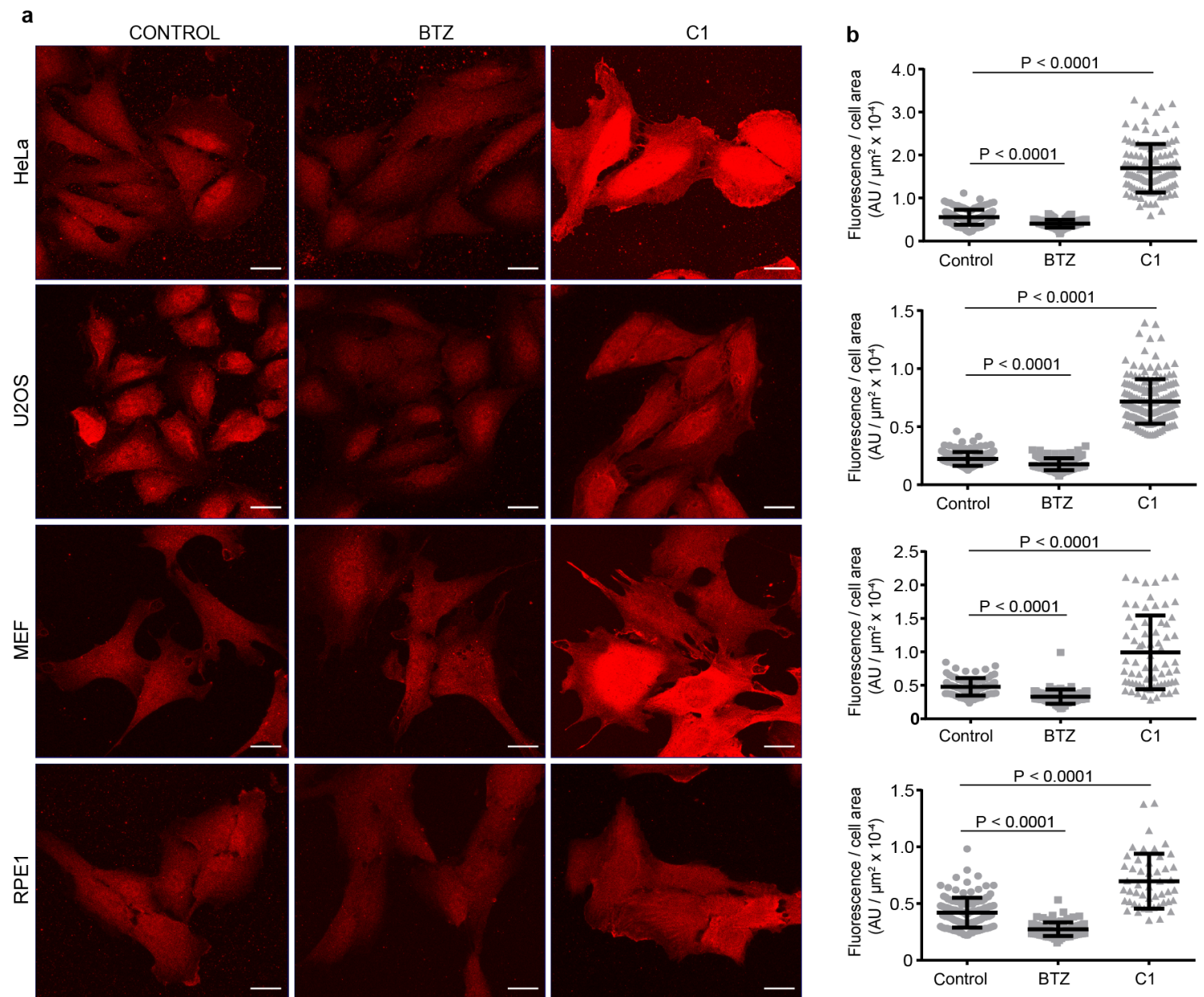

C

\begin{tabular}{cccc} 
Cell line & Control & BTZ & C1 \\
\hline HeLa & $5552 \pm 1731$ & $4043 \pm 893$ & $16936 \pm 5651$ \\
\hline U2OS & $2231 \pm 585$ & $1768 \pm 513$ & $7174 \pm 1914$ \\
\hline MEF & $4781 \pm 1307$ & $3463 \pm 1061$ & $9921 \pm 5514$ \\
\hline RPE1 & $4197 \pm 1313$ & $2737 \pm 611$ & $6974 \pm 2424$ \\
\hline
\end{tabular}

d

Fig. 4. Free Ub staining in HeLa, U2OS, MEF and RPE1 cells. a, Maximum projection images of free Ub staining with HA-tUI in HeLa, U2OS, MEF and RPE1 cells after $1 \mathrm{~h}$ incubation with $1 \mu \mathrm{M}$ proteasome inhibitor, BTZ or $10 \mu \mathrm{M}$ E1 inhibitor, C1. Scale bars, $20 \mu \mathrm{m}$. b, Mean fluorescence for HeLa, U2OS, MEF, and RPE1 cells after $1 \mathrm{~h}$ incubation with $1 \mu \mathrm{M}$ proteasome inhibitor or $10 \mu \mathrm{M} E 1$ inhibitor. AU, arbitrary units. Cells analyzed per condition: HeLa, control $n=131$, BTZ $n=125$, C1 $n=116$; U2OS, 
control $n=161, B T Z n=170, C 1 n=175 ; M E F$, control $n=80, B T Z n=79, C 1 n=69$; RPE1, control $n=133, B T Z n=87, C 1 n=49$. Bars show mean \pm s.d. Statistical analyses by unpaired Student's $t$-test with Welch's correction where appropriate. c, Relative free Ub from staining (mean fluorescence \pm s.d.) of untreated cells or after proteasome or E1 inhibition. d, Representative interphase (left panel) and mitotic (right panel) RPE-1 cells stained with HA-tUI (red) and DAPI (blue). Intensity measurements from 3D reconstructions employed Imaris software. Total cell fluorescence (arbitrary units; mean \pm s.d., $n \geq 3$ ) for interphase and mitotic RPE1 cells were $23.7 \pm 2.5$ and $38.4 \pm 3.5$, respectively, whereas fluorescence per unit volume was $134.1 \pm 8.6$ for interphase RPE1 cells and $463.2 \pm 70.6$ for mitotic RPE1 cells. Scale bars, $5 \mu \mathrm{m}$. 


\section{References}

$1 \quad$ Pickart, C. M. Back to the future with ubiquitin. Cell 116, 181-190 (2004).

2 Park, C. W. \& Ryu, K. Y. Cellular ubiquitin pool dynamics and homeostasis. BMB Rep 47, 475-482 (2014).

3 Ryu, K. Y., Garza, J. C., Lu, X. Y., Barsh, G. S. \& Kopito, R. R. Hypothalamic neurodegeneration and adult-onset obesity in mice lacking the Ubb polyubiquitin gene. Proc Natl Acad Sci U S A 105, 4016-4021, doi:10.1073/pnas.0800096105 (2008).

$4 \quad$ Ryu, K. Y. et al. The mouse polyubiquitin gene UbC is essential for fetal liver development, cellcycle progression and stress tolerance. EMBO J 26, 2693-2706, doi:10.1038/sj.emboj.7601722 (2007).

5 Ryu, K. Y. et al. The mouse polyubiquitin gene Ubb is essential for meiotic progression. Mol Cell Biol 28, 1136-1146, doi:10.1128/MCB.01566-07 (2008).

6 Kimura, Y. et al. An inhibitor of a deubiquitinating enzyme regulates ubiquitin homeostasis. Cell 137, 549-559, doi:10.1016/j.cell.2009.02.028 (2009).

7 Wang, C. H. et al. USP5/Leon deubiquitinase confines postsynaptic growth by maintaining ubiquitin homeostasis through Ubiquilin. Elife 6, doi:10.7554/eLife.26886 (2017).

Crimmins, S. et al. Transgenic rescue of ataxia mice with neuronal-specific expression of ubiquitin-specific protease 14. J Neurosci 26, 11423-11431, doi:10.1523/JNEUROSCI.360006.2006 (2006).

Chen, P. C. et al. The proteasome-associated deubiquitinating enzyme Usp14 is essential for the maintenance of synaptic ubiquitin levels and the development of neuromuscular junctions. $J$ Neurosci 29, 10909-10919, doi:10.1523/JNEUROSCI.2635-09.2009 (2009).

10 Oh, C., Park, S., Lee, E. K. \& Yoo, Y. J. Downregulation of ubiquitin level via knockdown of polyubiquitin gene Ubb as potential cancer therapeutic intervention. Sci Rep 3, 2623, doi:10.1038/srep02623 (2013).

11 Kedves, A. T. et al. Recurrent ubiquitin B silencing in gynecological cancers establishes dependence on ubiquitin C. J Clin Invest 127, 4554-4568, doi:10.1172/JCI92914 (2017).

12 Hallengren, J., Chen, P. C. \& Wilson, S. M. Neuronal ubiquitin homeostasis. Cell Biochem Biophys 67, 67-73, doi:10.1007/s12013-013-9634-4 (2013).

13 Dantuma, N. P., Groothuis, T. A., Salomons, F. A. \& Neefjes, J. A dynamic ubiquitin equilibrium couples proteasomal activity to chromatin remodeling. J Cell Biol 173, 19-26, doi:10.1083/jcb.200510071 (2006).

14 Yau, R. \& Rape, M. The increasing complexity of the ubiquitin code. Nat Cell Biol 18, 579-586, doi:10.1038/ncb3358 (2016).

15 Kaiser, S. E. et al. Protein standard absolute quantification (PSAQ) method for the measurement of cellular ubiquitin pools. Nat Methods 8, 691-696, doi:10.1038/nmeth.1649 (2011).

16 Reyes-Turcu, F. E. et al. The ubiquitin binding domain ZnF UBP recognizes the C-terminal diglycine motif of unanchored ubiquitin. Cell 124, 1197-1208, doi:10.1016/j.cell.2006.02.038 (2006).

17 Swanson, K. A., Kang, R. S., Stamenova, S. D., Hicke, L. \& Radhakrishnan, I. Solution structure of Vps27 UIM-ubiquitin complex important for endosomal sorting and receptor downregulation. EMBO J 22, 4597-4606, doi:10.1093/emboj/cdg471 (2003).

18 Lee, S. et al. Structural basis for ubiquitin recognition and autoubiquitination by Rabex-5. Nat Struct Mol Biol 13, 264-271, doi:10.1038/nsmb1064 (2006).

19 Penengo, L. et al. Crystal structure of the ubiquitin binding domains of rabex-5 reveals two modes of interaction with ubiquitin. Cell 124, 1183-1195, doi:10.1016/j.cell.2006.02.020 (2006). 
Maupin-Furlow, J. A. Ubiquitin-like proteins and their roles in archaea. Trends Microbiol 21, 3138, doi:10.1016/j.tim.2012.09.006 (2013).

Choi, Y. S., Jeon, Y. H., Ryu, K. S. \& Cheong, C. 60th residues of ubiquitin and Nedd8 are located out of E2-binding surfaces, but are important for K48 ubiquitin-linkage. FEBS Lett 583, 33233328, doi:10.1016/j.febslet.2009.09.034 (2009).

22 Zhang, D., Raasi, S. \& Fushman, D. Affinity makes the difference: nonselective interaction of the UBA domain of Ubiquilin-1 with monomeric ubiquitin and polyubiquitin chains. $J \mathrm{Mol}$ Biol 377, 162-180, doi:10.1016/j.jmb.2007.12.029 (2008).

23 Sokratous, K. et al. Probing affinity and ubiquitin linkage selectivity of ubiquitin-binding domains using mass spectrometry. J Am Chem Soc 134, 6416-6424, doi:10.1021/ja300749d (2012).

24 Swaney, D. L., Rodriguez-Mias, R. A. \& Villen, J. Phosphorylation of ubiquitin at Ser65 affects its polymerization, targets, and proteome-wide turnover. EMBO Rep 16, 1131-1144, doi:10.15252/embr.201540298 (2015).

25 Kane, L. A. et al. PINK1 phosphorylates ubiquitin to activate Parkin E3 ubiquitin ligase activity. J Cell Biol 205, 143-153, doi:10.1083/jcb.201402104 (2014).

26 Ordureau, A. et al. Defining roles of PARKIN and ubiquitin phosphorylation by PINK1 in mitochondrial quality control using a ubiquitin replacement strategy. Proc Natl Acad Sci U S A 112, 6637-6642, doi:10.1073/pnas.1506593112 (2015).

27 Koyano, F. et al. Ubiquitin is phosphorylated by PINK1 to activate parkin. Nature 510, 162-166, doi:10.1038/nature13392 (2014).

28 Harper, J. W., Ordureau, A. \& Heo, J. M. Building and decoding ubiquitin chains for mitophagy. Nat Rev Mol Cell Biol 19, 93-108, doi:10.1038/nrm.2017.129 (2018).

29 Wauer, T. et al. Ubiquitin Ser65 phosphorylation affects ubiquitin structure, chain assembly and hydrolysis. EMBO J 34, 307-325, doi:10.15252/embj.201489847 (2015).

30 Dang, L. C., Melandri, F. D. \& Stein, R. L. Kinetic and mechanistic studies on the hydrolysis of ubiquitin C-terminal 7-amido-4-methylcoumarin by deubiquitinating enzymes. Biochemistry 37, 1868-1879, doi:10.1021/bi9723360 (1998).

31 Geurink, P. P. et al. Development of Diubiquitin-Based FRET Probes To Quantify Ubiquitin Linkage Specificity of Deubiquitinating Enzymes. Chembiochem 17, 816-820, doi:10.1002/cbic.201600017 (2016).

32 Yao, T. \& Cohen, R. E. Ubiquitin-ovomucoid fusion proteins as model substrates for monitoring degradation and deubiquitination by proteasomes. Methods Enzymol 398, 522-540, doi:10.1016/S0076-6879(05)98043-9 (2005).

33 Wang, T. et al. Evidence for bidentate substrate binding as the basis for the K48 linkage specificity of otubain 1. J Mol Biol 386, 1011-1023, doi:10.1016/j.jmb.2008.12.085 (2009).

34 Wiener, R. et al. E2 ubiquitin-conjugating enzymes regulate the deubiquitinating activity of OTUB1. Nat Struct Mol Biol 20, 1033-1039, doi:10.1038/nsmb.2655 (2013).

35 Gates, Z. P., Stephan, J. R., Lee, D. J. \& Kent, S. B. Rapid formal hydrolysis of peptidealphathioesters. Chem Commun (Camb) 49, 786-788, doi:10.1039/c2cc38229f (2013).

36 Shahnawaz, M., Thapa, A. \& Park, I. S. Stable activity of a deubiquitylating enzyme (Usp2-cc) in the presence of high concentrations of urea and its application to purify aggregation-prone peptides. Biochem Biophys Res Commun 359, 801-805, doi:10.1016/j.bbrc.2007.05.186 (2007). Cell 44, 325-340, doi:10.1016/j.molcel.2011.08.025 (2011). by mass spectrometry. Cell Biochem Biophys 67, 139-147, doi:10.1007/s12013-013-9625-5 (2013). 
Chen, J. J. et al. Mechanistic studies of substrate-assisted inhibition of ubiquitin-activating enzyme by adenosine sulfamate analogues. I Biol Chem 286, 40867-40877, doi:10.1074/jbc.M111.279984 (2011).

40 Bianchi, M. et al. Dynamic transcription of ubiquitin genes under basal and stressful conditions and new insights into the multiple UBC transcript variants. Gene 573, 100-109, doi:10.1016/j.gene.2015.07.030 (2015).

41 Liu, Z. et al. Noncovalent dimerization of ubiquitin. Angew Chem Int Ed Engl 51, 469-472, doi:10.1002/anie.201106190 (2012).

42 Joo, H. Y. et al. Regulation of cell cycle progression and gene expression by $\mathrm{H} 2 \mathrm{~A}$ deubiquitination. Nature 449, 1068-1072, doi:10.1038/nature06256 (2007).

43 Jencks, W. P. On the attribution and additivity of binding energies. Proc Natl Acad Sci U S A 78, 4046-4050 (1981).

44 Scott, D., Oldham, N. J., Strachan, J., Searle, M. S. \& Layfield, R. Ubiquitin-binding domains: mechanisms of ubiquitin recognition and use as tools to investigate ubiquitin-modified proteomes. Proteomics 15, 844-861, doi:10.1002/pmic.201400341 (2015).

45 Hicke, L., Schubert, H. L. \& Hill, C. P. Ubiquitin-binding domains. Nat Rev Mol Cell Biol 6, 610-621, doi:10.1038/nrm1701 (2005).

46 Morrow, M. E. et al. Active site alanine mutations convert deubiquitinases into high-affinity ubiquitin-binding proteins. EMBO Rep 19, doi:10.15252/embr.201745680 (2018).

47 Harrigan, J. A., Jacq, X., Martin, N. M. \& Jackson, S. P. Deubiquitylating enzymes and drug discovery: emerging opportunities. Nat Rev Drug Discov 17, 57-78, doi:10.1038/nrd.2017.152 (2018).

48 Majetschak, M. Extracellular ubiquitin: immune modulator and endogenous opponent of damage-associated molecular pattern molecules. J Leukoc Biol 89, 205-219, doi:10.1189/jlb.0510316 (2011).

49 Raasi, S. \& Pickart, C. M. Ubiquitin chain synthesis. Methods Mol Biol 301, 47-55, doi:10.1385/159259-895-1:047 (2005).

50 Whitby, F. G., Xia, G., Pickart, C. M. \& Hill, C. P. Crystal structure of the human ubiquitin-like protein NEDD8 and interactions with ubiquitin pathway enzymes. J Biol Chem 273, 34983-34991 (1998).

51 Wilkinson, K. D. et al. Metabolism of the polyubiquitin degradation signal: structure, mechanism, and role of isopeptidase T. Biochemistry 34, 14535-14546 (1995).

52 Wilkinson, K. D. Quantitative analysis of protein-protein interactions. Methods Mol Biol 261, 1532, doi:10.1385/1-59259-762-9:015 (2004). 


\section{Acknowledgements}

We thank O. Peersen for assistance with the rapid-kinetics experiments and use of the Bio-Logic stopped-flow spectrofluorometer, C. Wolberger for OTUB1 protein, B. Brasher for phosphoubiquitin, and R. Handa for use of the Imaris image analysis software. This research was supported by NIH-NIGMS grant R01 GM37666 (to R.E.C.) and NIHNIEHS grant R21 ES029150 (to R.E.C. and T.Y.).

\section{Author contributions}

Y.C. and R.E.C. conceived and designed the ubiquitin sensor reagents, and Y.C. produced the sensors and characterized them in vitro. S.B., T.Y., and R.E.C. conceived of the cell-based studies, which were done by S.B., L.P., and F.S. All authors contributed to writing or commenting on the manuscript.

\section{Competing interests}

U.S. patent no. 10,018,634 has been awarded to Colorado State University Research Foundation (R.E.C. and Y.C., inventors) for ubiquitin sensors and assays described in this paper. 